\title{
Some remarks about flux linkage and inductance
}

\author{
S. Kurz \\ University of the Federal Armed Forces, School of Electrical Engineering, Holstenhofweg 85, 22043 Hamburg, Germany
}

\begin{abstract}
In the area of computational electromagnetics there is an increasing demand for various coupled simulations. One example is the coupling between field and circuit simulation for the description of electromagnetic devices. In the context of such couplings, theoretical questions arise as well. How can a field device be represented as an equivalent multiport circuit element? What is meant by flux linkage if the considered conductors are not filamentary? What is meant by inductance if the magnetic media exhibit nonlinear behaviour? These questions and their answers are not new. However, according to the author's view, these issues are not sufficiently addressed in the usual textbooks. The aim of the paper is therefore to (hopefully) answer the questions concisely and correctly. The modern language of differential forms will be employed for this purpose.
\end{abstract}

\section{Introduction}

To couple field and circuit descriptions of electromagnetic devices the terminal currents and terminal voltages must be properly defined. In the absence of displacement currents the terminal current of a circuit is well defined by the contour integral of the magnetic field. However, it is not so clear how to define the terminal voltage in a time-dependent situation, since the electric field is in general not conservative. This fact gives rise to an implicit limitation on the use of voltmeters. All voltmeters are designed to measure the line integral of the electric field along the path formed by the connecting leads. Therefore the voltage between two points has a meaning only when the path of integration is contained in a region of space in which the electric field behaves approximately as an electrostatic field (Fano et al., 1960, p. 264). These considerations will be formalised in Sect. 2 and lead to the concept of the Electromagnetic Circuit Element (ECE).

In Sect. 3, the textbook definition of flux linkage is extended beyond the case of filamentary conductors. It will be shown that each pair of an ECE's terminals associated with a

Correspondence to: S. Kurz

(stefan.kurz@unibw-hamburg.de) conducting path can be assigned a flux linkage. The terminal voltages can then be related to the terminal currents by a DC resistance matrix plus the time derivative of the linked fluxes.

Electromagnetic devices often exhibit non-linear behaviour due to iron saturation. The usual definition of inductance coefficients breaks down in such situations, because it crucially relies on the principle of linear superposition. Section 4 is devoted to the question how inductance coefficients can be defined in a way that also accounts for non-linearities.

The modern language of differential forms will be used throughout this paper. We denote by $\mathcal{F}^{p}(\Omega)$ the set of differential $p$-forms over a domain $\Omega$ and represent the electromagnetic field quantities by differential forms of various degree. The electric scalar potential $\varphi \in \mathcal{F}^{0}(\Omega)$ can be seen as a 0 -form. The electric field $\underline{E} \in \mathcal{F}^{-1}(\Omega)$, the magnetic field $\underline{H} \in \mathcal{F}^{1}(\Omega)$ and the magnetic vector potential $\underline{A} \in \mathcal{F}^{1}(\Omega)$ are all represented by 1 -forms. The electric and magnetic flux densities $\underline{D} \in \mathcal{F}^{2}(\Omega)$ and $\underline{B} \in \mathcal{F}^{2}(\Omega)$, respectively, are regarded as 2-forms, while the electric charge density $\rho \in \mathcal{F}^{3}(\Omega)$ is represented by a 3 -form. Boundary traces of the fields can be obtained by means of the trace operator $t$. All spatial derivatives are expressed in terms of the exterior derivative $\mathrm{d}$, while $\partial_{t}$ denotes the time derivative. Trace and derivative operators commute with each other.

\section{Electromagnetic circuit elements}

Under which conditions does an electromagnetic device provided with terminals behave like a circuit?

To answer this question and to precisely define terminal currents and voltages we introduce the concept of the Electromagnetic Circuit Element (ECE), see Munteanu and Ioan (2001) and the references cited therein. An ECE is a domain $\Omega$ whose boundary $\Gamma$ is equipped with $n$ ideal terminal connectors $S_{1}, S_{2}, \ldots, S_{n}$. The terminal connectors and the complementary insulating surface $S_{\mathrm{e}}$ form a disjoint partition of $\Gamma$,

$$
\Gamma=\partial \Omega=S_{\mathrm{e}}+\sum_{k=1}^{n} S_{k},
$$




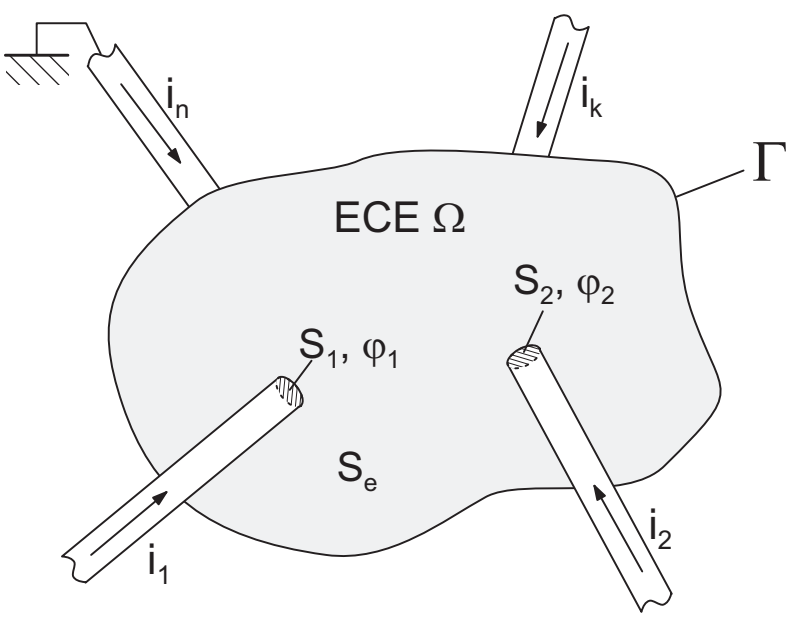

Fig. 1. An Electromagnetic Circuit Element (ECE) is a domain $\Omega$ whose boundary $\Gamma$ is equipped with $n$ ideal terminal connectors $S_{1}, S_{2}, \ldots, S_{n}$. Current can only flow through these connectors, but not through the insulating surface $S_{\mathrm{e}}$. Moreover, it is required that there is no magnetic coupling through the boundary.

where $\partial$ denotes the boundary operator. To avoid topological difficulties, we assume that the domain $\Omega$ is star-shaped. For an analysis of the general case see Kettunen (2001).

The defining conditions for the ECE refer only to the boundary of the domain occupied by the device, and not to its internal structure. There are three boundary conditions,

$$
\begin{gathered}
\exists \underline{\varphi}_{\Gamma} \in \mathcal{F}^{0}(\Gamma): \mathbf{t} \underline{E}=-\mathrm{d} \underline{\varphi}_{\Gamma}, \\
\underline{\varphi}_{\Gamma}=\underline{\varphi}_{k}=\mathrm{const} \quad \text { on } S_{k}, \\
\mathbf{t d} \underline{H}=\mathbf{t}\left(\underline{J}+\partial_{t} \underline{D}\right)=0 \quad \text { on } S_{\mathrm{e}} .
\end{gathered}
$$

The first condition means that the tangential component $\mathbf{t} \underline{E}$ of the electric field at the boundary can be represented as the surface gradient of a surface potential $\underline{\varphi}_{\Gamma}$. Condition (2a) forbids magnetic coupling between the interior and the exterior of the ECE. In fact,

$$
\mathbf{t} \partial_{t} \underline{B}=-\mathbf{t d} \underline{E}=-\mathrm{d} \underline{\mathbf{t}} \underline{E}=\operatorname{dd} \underline{\varphi}_{\Gamma}=0,
$$

so no time-dependent flux can evade the domain. The only interaction of the ECE with its exterior is accomplished through its terminals. The second condition (2b) imposes equipotentiality of the terminals. Finally, Eq. (2c) implies that (conduction or displacement) current can only flow through the terminals.

Let us define terminal currents $i_{k}$ and voltages $u_{k}$,

$$
\begin{aligned}
& i_{k}=-\int_{S_{k}} \mathbf{t}\left(\underline{J}+\partial_{t} \underline{D}\right)=-\int_{\partial S_{k}} \underline{\mathbf{t}} \underline{H}, \\
& u_{k}=\underline{\varphi}_{k}-\underline{\varphi}_{n}, \quad k=1, \ldots, n .
\end{aligned}
$$

An ECE with $n$ terminals can be considered a system with $n-$ 1 inputs, namely currents $i_{k}$ or voltages $u_{k}$, and $n-1$ outputs - those currents and voltages which are not input quantities.
The terminal $S_{n}$ has been chosen as ground terminal, $u_{n}=0$. The input-output relation is, for a given initial state, uniquely determined. Despite the finite number of inputs and outputs, the system has an infinite dimension of the state space.

Kirchhoff's current law is automatically satisfied, since

$$
\begin{aligned}
\sum_{k=1}^{n} i_{k} & =-\sum_{k=1}^{n} \int_{\partial S_{k}} \mathbf{t} \underline{H}=-\sum_{k=1}^{n} \int_{S_{k}} \mathbf{t d} \underline{H} \\
& =-\int_{\Gamma=\partial \Omega} \mathbf{t d} \underline{H}=-\int_{\Omega} \operatorname{tdd} \underline{H}=0 .
\end{aligned}
$$

Kirchhoff's voltage law holds trivially, due to the definition (Eq. 5). The validity of Kirchhoff's laws ensures that the ECE can be coupled with external circuitry as if it was yet another circuit. The power received by the ECE from some exterior circuit can be expressed similarly to the case of classical circuits, as the sum of voltage-current products for all terminals,

$$
\begin{aligned}
p_{\Gamma}(t) & =-\int_{\Gamma} \mathbf{t}(\underline{E} \wedge \underline{H})=\int_{\Gamma} \mathrm{d} \underline{\varphi}_{\Gamma} \wedge \underline{\mathbf{t}} \underline{H} \\
& =-\int_{\Gamma} \underline{\varphi}_{\Gamma} \wedge \mathbf{t d} \underline{H}=-\sum_{k=1}^{n} \int_{S_{k}} \underline{\varphi}_{\Gamma} \wedge \mathbf{t d} \underline{H} \\
& =-\underline{\varphi}_{k} \sum_{k=1}^{n} \int_{S_{k}} \mathbf{t d} \underline{H}=\sum_{k=1}^{n} \underline{\varphi}_{k} i_{k} \\
& =\sum_{k=1}^{n-1}\left(\underline{\varphi}_{k}-\underline{\varphi}_{n}\right) i_{k}+\underline{\varphi}_{n} \sum_{k=1}^{n} i_{k}=\sum_{k=1}^{n-1} u_{k} i_{k} .
\end{aligned}
$$

The ECE boundary conditions are, of course, satisfied only approximately in practice, and the difference

$$
\varepsilon=\int_{\Gamma} \mathbf{t}(\underline{E} \wedge \underline{H})+\sum_{k=1}^{n-1} u_{k} i_{k}
$$

of both expressions for the power gives a measure of the approximation error. If this error is unacceptably high, one or more of the conditions (2) are not fulfilled and the domain $\Omega$ cannot be considered as a circuit element.

\section{Flux linkage}

The concept of an ECE allowed a precise definition of terminal currents and voltages. As a next step, we will proceed to the notion of flux linkage. To this end, we consider an ECE that represents a quasi-stationary magnetic field system. The fields in $\Omega$ shall be therefore governed by Maxwell's equations in the form

$$
\begin{aligned}
\mathrm{d} \underline{H} & =\underline{J}, \\
\mathrm{~d} \underline{E} & =-\partial_{t} \underline{B}, \\
\mathrm{~d} \underline{B} & =0 .
\end{aligned}
$$

Maxwell's equations have to be complemented by material laws. We denote the conducting subdomain by $\Omega_{\sigma}$, i.e.

$$
\sigma>0 \quad \text { in } \Omega_{\sigma} \subset \Omega, \quad \sigma=0 \quad \text { in } \Omega \backslash \Omega_{\sigma},
$$


where $\sigma$ is the electrical conductivity. We assume that a linear Ohm's law holds in $\Omega_{\sigma}$, which can be stated locally as

$$
\underline{J}=*_{\sigma} \underline{E} .
$$

Herein, $*_{\sigma}$ denotes the Hodge operator that is adapted to the conductivity distribution. In vector notation, Eq. (11) just means $\boldsymbol{J}=\sigma \boldsymbol{E}$.

Finally, we assume that each terminal connector $S_{k}$, $k=1, \ldots, n-1$ is connected to the ground terminal $S_{n}$ by a path which is completely contained in $\Omega_{\sigma}$. This condition restricts the topology of $\Omega_{\sigma}$ in order to simplify the subsequent derivations. The restriction could be lifted without significantly affecting the main results. For a detailed discussion about the topological aspects see S. Suuriniemi and Kettunen (2003).

We don't give a specific relation between $\underline{B}$ and $\underline{H}$, but have in mind some rather general non-linear magnetic material behaviour, with a sufficient degree of regularity, Hantila (1974).

Since the domain $\Omega$ is star-shaped, the fields in $\Omega$ can be represented without topological difficulties by means of the potentials,

$$
\underline{B}=\mathrm{d} \underline{A}, \quad \underline{E}=-\mathrm{d} \varphi-\partial_{t} \underline{A} .
$$

In $\Omega$, the line integral of the electric field is in general dependent on the path of integration. If we relate the surface potential $\varphi_{\Gamma} \in \mathcal{F}^{0}(\Gamma)$ to the potential $\varphi \in \mathcal{F}^{0}(\Omega)$ by

$$
\underline{\varphi}_{\Gamma}=\mathbf{t} \underline{\varphi},
$$

then the magnetic vector potential has to obey the boundary condition

$$
\partial_{t} \underline{\mathbf{t}} \underline{A}=-\mathrm{d} \mathbf{t} \varphi-\mathbf{t} \underline{E}=-\mathrm{d} \varphi_{\Gamma}-\mathbf{t} \underline{E}=0 .
$$

We consider two different states (say 1 and 2) of the system, and find

$$
\begin{aligned}
\mathrm{d}\left(\underline{\varphi}_{1} \wedge \underline{J}_{2}\right) & =\underline{\varphi}_{1} \wedge \mathrm{d} \underline{J}_{2}+\left(\mathrm{d} \underline{\varphi}_{1}\right) \wedge \underline{J}_{2} \\
& =-\left(\underline{E}_{1}+\partial_{t} \underline{A}_{1}\right) \wedge \underline{J}_{2} .
\end{aligned}
$$

The first term drops out due to the solenoidality $\mathrm{d} \underline{J}=0$ of the current density. We integrate Eq. (15) over $\Omega$ and use Stokes' theorem to obtain

$$
\int_{\Gamma} \underline{t}_{1} \wedge \underline{\mathbf{t}}_{2}=-\int_{\Omega}\left(\underline{E}_{1}+\partial_{t} \underline{A}_{1}\right) \wedge \underline{J}_{2} .
$$

Proceeding along the same lines as in Eq. (7) reveals

$$
\int_{\Gamma} \underline{\mathbf{t}}_{1} \wedge \underline{\mathbf{t}}_{2}=\int_{\Gamma} \underline{\varphi}_{\Gamma, 1} \wedge \mathbf{t} \mathrm{d} \underline{H}_{2}=-\sum_{k=1}^{n-1} u_{1, k} i_{2, k} .
$$

We introduce the vector of currents $\mathbf{i}=\left(i_{1}, \ldots, i_{n-1}\right)^{T} \in$ $\mathbb{R}^{n-1}$ and the vector of voltages $\mathbf{u}=\left(u_{1}, \ldots, u_{n-1}\right)^{T} \in \mathbb{R}^{n-1}$. From Eqs. (16) and (17) we receive

$$
\mathbf{u}_{1}^{T} \mathbf{i}_{2}=\int_{\Omega}\left(\underline{E}_{1}+\partial_{t} \underline{A}_{1}\right) \wedge \underline{J}_{2} .
$$

Since Eq. (18) holds for arbitrary states 1 and 2 of the system, we may equally well swap the indices and subtract the resulting equations from each other, yielding

$$
\begin{aligned}
\mathbf{u}_{1}^{T} \mathbf{i}_{2}-\mathbf{u}_{2}^{T} \mathbf{i}_{1} & =\int_{\Omega} \underline{E}_{1} \wedge \underline{J}_{2}-\underline{E}_{2} \wedge \underline{J}_{1} \\
& +\int_{\Omega} \partial_{t} \underline{A}_{1} \wedge \underline{J}_{2}-\partial_{t} \underline{A}_{2} \wedge \underline{J}_{1} .
\end{aligned}
$$

Since Ohm's law (11) induces a symmetric bilinear form,

$$
\underline{E}_{1} \wedge \underline{J}_{2}=\underline{E}_{1} \wedge\left(*_{\sigma} \underline{E}_{2}\right)=\underline{E}_{2} \wedge\left(*_{\sigma} \underline{E}_{1}\right)=\underline{E}_{2} \wedge \underline{J}_{1},
$$

the first integral in Eq. (19) drops out and we are left with the reciprocity theorem

$$
\mathbf{u}_{1}^{T} \mathbf{i}_{2}-\mathbf{u}_{2}^{T} \mathbf{i}_{1}=\int_{\Omega} \partial_{t} \underline{A}_{1} \wedge \underline{J}_{2}-\partial_{t} \underline{A}_{2} \wedge \underline{J}_{1} .
$$

Let us in a first step consider stationary DC states, $\partial_{t}=0$. DC currents and voltages shall be denoted by capital letters. By exploiting once again the linearity of Ohm's law, we are able to express the current density $\underline{J}$ as a multilinear function of the terminal currents,

$$
\underline{J}=\sum_{k=1}^{n-1} \underline{\tau}_{k} I_{k}=\underline{\boldsymbol{\tau}}^{T} \mathbf{I}
$$

Note that $\underline{\tau}_{k} \in \mathcal{F}^{2}(\Omega)$ is the current density in the system when the DC current through terminal $S_{k}$ equals 1 Amp and all other currents equal zero. The current will return through the ground terminal $S_{n}$, which is ensured by the topological prerequisite about $\Omega_{\sigma}$. From Eqs. (11), (18), (22), and the property $*_{\sigma} *_{\sigma}=1$ we find

$$
\begin{aligned}
\mathbf{U}_{1}^{T} \mathbf{I}_{2} & =\int_{\Omega_{\sigma}} \underline{E}_{1} \wedge \underline{J}_{2}=\int_{\Omega_{\sigma}} *_{\sigma} \underline{J}_{1} \wedge \underline{J}_{2} \\
& =\mathbf{I}_{1}^{T}\left(\int_{\Omega_{\sigma}} *_{\sigma} \underline{\boldsymbol{\tau}} \wedge \underline{\boldsymbol{\tau}}^{T}\right) \mathbf{I}_{2} .
\end{aligned}
$$

We define the resistance matrix $\mathbf{R} \in \mathbb{R}^{(n-1) \times(n-1)}$ according to

$$
\mathbf{R}=\int_{\Omega_{\sigma}} *_{\sigma} \underline{\boldsymbol{\tau}} \wedge \underline{\boldsymbol{\tau}}^{T}
$$

where $\mathbf{R}=\mathbf{R}^{T}$. Since Eq. (23) holds for arbitrary DC states $\mathbf{I}_{2}$, we can conclude

$$
\mathbf{U}=\mathbf{R} \mathbf{I}
$$

Armed with these results we proceed to a more general consideration, where state 1 is an arbitrary state (no index), while state 2 is a stationary DC state (index 0). In this case, Eq. (21) reduces to

$$
\mathbf{u}^{T} \mathbf{I}_{0}-\mathbf{U}_{0}^{T} \mathbf{i}=\int_{\Omega_{\sigma}} \partial_{t} \underline{A} \wedge \underline{J}_{0}
$$




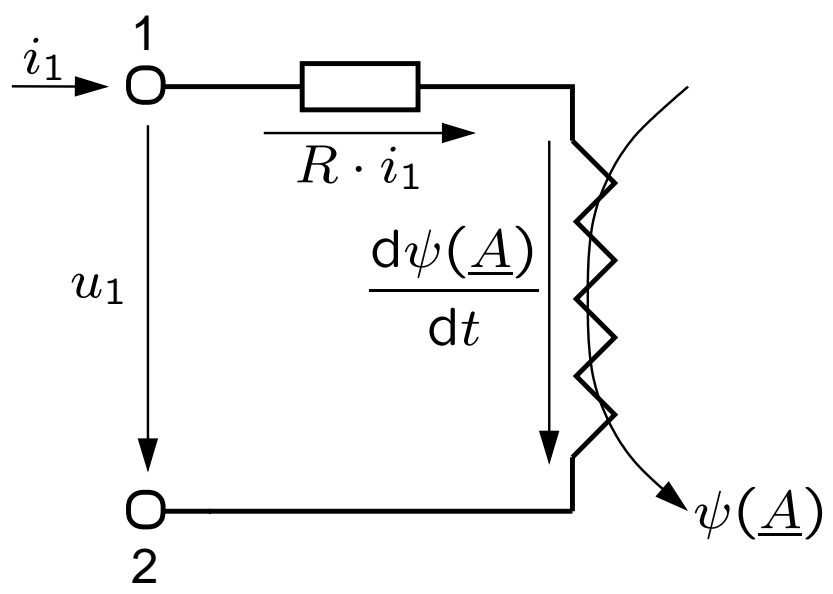

Fig. 2. Equivalent circuit diagram for a simple ECE with two terminals. The ECE represents a quasi-stationary magnetic field system.

It is sufficient to restrict the integration over the conducting subdomain $\Omega_{\sigma}$. By using Eqs. (22) and (25) we find

$$
\mathbf{u}^{T} \mathbf{I}_{0}=\left(\mathbf{i}^{T} \mathbf{R}+\int_{\Omega_{\sigma}} \partial_{t} \underline{A} \wedge \underline{\boldsymbol{\tau}}^{T}\right) \mathbf{I}_{0} .
$$

Since this holds for arbitrary DC states $\mathbf{I}_{0}$, we conclude

$$
\mathbf{u}=\mathbf{R} \mathbf{i}+\int_{\Omega_{\sigma}} \partial_{t} \underline{A} \wedge \underline{\boldsymbol{\tau}}=\mathbf{R} \mathbf{i}+\partial_{t} \boldsymbol{\psi}(\underline{A})
$$

with the generalised flux linkage

$$
\psi: \mathcal{F}^{1}(\Omega) \rightarrow \mathbb{R}^{n-1}: \underline{A} \mapsto \psi(\underline{A})=\int_{\Omega_{\sigma}} \underline{A} \wedge \underline{\boldsymbol{\tau}} .
$$

This is the main result of the section. For the case of a simple ECE with $n=2$ terminals, Eq. (28) reads

$$
u_{1}=R i_{1}+\partial_{t} \psi(\underline{A}), \quad \psi(\underline{A})=\int_{\Omega_{\sigma}} \underline{A} \wedge \underline{\tau},
$$

and can be represented by an equivalent circuit diagram as shown in Fig. 2.

Equation (28) completely determines the terminal behaviour of the ECE and can be used as a starting point for the coupling of field and circuit descriptions. Be aware that Eq. (28) does not relate to a decomposition of the received power (Eq. 7) into Ohm's losses, and power converted into field energy. The second term may relate to losses as well. Think of Fig. 2 as the equivalent circuit diagram of a shortcircuited transformer. In this case $R$ reflects the resistance of the primary winding. The dissipated power in the secondary winding is transmitted via the field and contained in the term $i \partial_{t} \psi(\underline{A})$.

The evaluation of the flux linkage (Eq. 29) becomes straightforward if we have to deal with filamentary conductors, because in this case the filaments define the paths of the current. For a single filament $C$ which bounds a surface $F$ we have $\underline{\tau}=\underline{\delta}_{C}$, where $\underline{\delta}_{C}$ is a singular 2-form which is localised along the contour $C$. The coefficients of such forms can be represented in terms of $\delta$-distributions. For sufficiently smooth 1 -forms $\omega$ we have

$$
\int_{\Omega} \omega \wedge \underline{\delta}_{C}=\int_{C} \omega
$$

The expression (29) for the flux linkage therefore reduces to

$$
\psi(\underline{A})=\int_{\Omega} \underline{A} \wedge \underline{\delta}_{C}=\int_{C} \underline{A}=\int_{F} \underline{B} .
$$

Equation (32) is of course the usual textbook definition of the flux linkage. For obvious reasons, mappings $\mathcal{F}^{p}(\Omega) \rightarrow \mathbb{R}$ are called de Rham's currents, see de Rham (1984).

\section{Inductance and energy}

The definition of inductances will allow us to set up a finitedimensional state space model of the system. Since the ECE considered so far has an infinite dimension of the state space, we can conclude that it is not possible to define inductances without further restrictions.

Suppose that Eq. (22) is valid not only for DC currents, but even for all time-dependent currents,

$$
\underline{J}=\sum_{k=1}^{n-1} \underline{\tau}_{k} i_{k}=\underline{\boldsymbol{\tau}}^{T} \mathbf{i}
$$

This can be enforced by requiring that all conductors are of the filamentary type and that $\Omega_{\sigma}$ contains no loops. The latter condition eliminates situations such as the short-circuited transformer, whose secondary winding would be otherwise invisible from a DC point of view.

Then, given a state $\mathbf{i}$, the current density $\underline{J}(\mathbf{i})$ is uniquely determined by Eq. (33). This in turn determines a unique vector potential $\underline{A}(\mathbf{i})$, flux density $\underline{B}(\mathbf{i})$ and field $\underline{H}(\mathbf{i})$, provided there is no hysteresis. These quantities depend on $\mathbf{i}$, but not on the rate of change of $\mathbf{i}$. They will be subsequently regarded as forms defined on $\Omega \times \mathbb{R}^{n-1}$. This is reflected in the notation by explicitely writing the argument $\mathbf{i}$. The exterior derivative w.r.t. the state space $\mathbb{R}^{n-1}$ will be denoted by $\delta$.

Plugging the vector potential $\underline{A(i)}$ into Eq. (29) yields the flux mapping

$$
\begin{gathered}
\tilde{\boldsymbol{\psi}}: \mathbb{R}^{n-1} \rightarrow \mathbb{R}^{n-1}: \\
\mathbf{i} \mapsto \tilde{\boldsymbol{\psi}}(\mathbf{i})=\boldsymbol{\psi}(\underline{A}(\mathbf{i}))=\int_{\Omega_{\sigma}} \underline{A}(\mathbf{i}) \wedge \underline{\boldsymbol{\tau}} .
\end{gathered}
$$

For the differential of the flux mapping we have by definition

$$
\delta \tilde{\boldsymbol{\psi}}(\mathbf{i})=\int_{\Omega_{\sigma}} \delta \underline{A}(\mathbf{i}) \wedge \underline{\boldsymbol{\tau}} \equiv \mathbf{L}(\mathbf{i}) \delta \mathbf{i},
$$

where the elements of $\delta \tilde{\boldsymbol{\psi}}$ (i) can be seen as 1-forms over the state space. It follows that

$$
\mathbf{L}(\mathbf{i})=\left(L_{l k}(\mathbf{i})\right)=\left(\frac{\partial \tilde{\psi}_{l}}{\partial i_{k}}\right) \quad \in \mathbb{R}^{(n-1) \times(n-1)} .
$$


The elements of the matrix (36) are called incremental inductances, Demerdash and Nehl (1999). If we consider a trajectory $\mathbf{i}(t)$ in state space, then the exterior derivative $\delta$ can be expressed by pullback as $\delta=\partial_{t} \wedge \mathrm{d} t$. From Eqs. (28) and (35) we thus derive

$$
\mathbf{u}=\mathbf{R} \mathbf{i}+\mathbf{L}(\mathbf{i}) \partial_{t} \mathbf{i}
$$

The incremental inductance matrix $\mathbf{L}(\mathbf{i})$ relates the time derivative of the currents to the induced voltages.

From Stokes' theorem in state space we conclude

$$
\int_{0}^{\mathbf{i}} \delta \tilde{\psi}(\mathbf{i})=\tilde{\psi}(\mathbf{i})-\tilde{\psi}(0)
$$

the integral being path independent. We take advantage of this freedom, and connect the points 0 and $\mathbf{i}$ in state space by a straight line. This results in

$$
\begin{aligned}
\tilde{\psi}(\mathbf{i}) & =\mathbf{L}^{\psi}(\mathbf{i}) \mathbf{i}+\tilde{\psi}(0), \\
\mathbf{L}^{\psi}(\mathbf{i}) & =\int_{0}^{1} \mathbf{L}(\lambda \mathbf{i}) \mathrm{d} \lambda .
\end{aligned}
$$

The elements of the matrix (40) are called apparent inductances, Demerdash and Nehl (1999). The apparent inductance matrix $\mathbf{L}^{\psi}$ (i) relates the currents to the flux linkages. Note that the usual definition of inductance coefficients $L_{l k}^{\psi}$ as "flux linkage of terminal \# $l$ divided by terminal current \# $k$ " breaks down in a non-linear situation, because it relies on the pinciple of linear superposition. For linear systems, $\mathbf{L}^{\psi}=\mathbf{L}$ follows immediately from Eq. (40).

To arrive at an expression for the energy, let us consider the 1 -form

$$
\begin{aligned}
\boldsymbol{\omega}=\mathbf{i}^{T} \delta \tilde{\boldsymbol{\psi}}(\mathbf{i}) & =\mathbf{i}^{T} \mathbf{L}(\mathbf{i}) \delta \mathbf{i}=\sum_{k=1}^{n-1} \omega_{k} \delta i_{k}, \\
\omega_{k} & =\sum_{l=1}^{n-1} i_{l} L_{l k}(\mathbf{i})
\end{aligned}
$$

$\omega \in \mathcal{F}^{1}\left(\mathbb{R}^{n-1}\right)$, and compute its exterior derivative

$$
\delta \boldsymbol{\omega}=\sum_{k=1}^{n-1} \sum_{m<k}\left(\frac{\partial \omega_{k}}{\partial i_{m}}-\frac{\partial \omega_{m}}{\partial i_{k}}\right) \delta i_{m} \wedge \delta i_{k}
$$

Taking into account

$$
\begin{aligned}
& \frac{\partial \omega_{k}}{\partial i_{m}}=L_{m k}+\sum_{l=1}^{n-1} i_{l} \frac{\partial L_{l k}}{\partial i_{m}}=L_{m k}+\sum_{l=1}^{n-1} i_{l} \frac{\partial^{2} \tilde{\psi}_{l}}{\partial i_{k} \partial i_{m}}, \\
& \frac{\partial \omega_{m}}{\partial i_{k}}=L_{k m}+\sum_{l=1}^{n-1} i_{l} \frac{\partial L_{l m}}{\partial i_{k}}=L_{k m}+\sum_{l=1}^{n-1} i_{l} \frac{\partial^{2} \tilde{\psi}_{l}}{\partial i_{m} \partial i_{k}},
\end{aligned}
$$

we obtain

$$
\delta \omega=\sum_{k=1}^{n-1} \sum_{m<k}\left(L_{m k}-L_{k m}\right) \delta i_{m} \wedge \delta i_{k}
$$

On the other hand, from Eqs. (33), (35) and (41) we receive

$$
\begin{aligned}
\boldsymbol{\omega} & =\mathbf{i}^{T} \int_{\Omega_{\sigma}} \delta \underline{A}(\mathbf{i}) \wedge \underline{\boldsymbol{\tau}}=\int_{\Omega_{\sigma}} \delta \underline{A}(\mathbf{i}) \wedge \underline{J}(\mathbf{i}) \\
& =\int_{\Omega} \delta \underline{A}(\mathbf{i}) \wedge \mathrm{d} \underline{H}(\mathbf{i}) \\
& =\int_{\Omega} \delta \mathrm{d} \underline{A}(\mathbf{i}) \wedge \underline{H}(\mathbf{i})-\int_{\Gamma} \delta \underline{\mathbf{t}} \underline{A}(\mathbf{i}) \wedge \underline{\mathbf{t}} \underline{H}(\mathbf{i}) \\
& =\int_{\Omega} \delta \underline{B}(\mathbf{i}) \wedge \underline{H}(\mathbf{i})=\delta W(\mathbf{i}) .
\end{aligned}
$$

The boundary integral in the second last line vanishes in the light of the boundary condition (Eq. 14). In Eq. (45), the magnetic field energy

$$
W: \mathbb{R}^{n-1} \rightarrow \mathbb{R}: \mathbf{i} \mapsto W(\mathbf{i})
$$

stored in the domain $\Omega$ has been introduced. We combine Eqs. (41) and (45) into

$$
\boldsymbol{\omega}=\mathbf{i}^{T} \mathbf{L}(\mathbf{i}) \delta \mathbf{i}=\delta W(\mathbf{i}) .
$$

Poincaré's lemma in connection with Eq. (44) yields

$$
0=\delta(\delta W(\mathbf{i}))=\delta \omega=\sum_{k=1}^{n-1} \sum_{m<k}\left(L_{m k}-L_{k m}\right) \delta i_{m} \wedge \delta i_{k},
$$

thus $\mathbf{L}=\mathbf{L}^{T}$. Not only the incremental inductance matrix, but also the apparent inductance matrix is symmetric, due to the definition (40). The symmetry of the inductance matrices turns out to be the integrability condition that connects the differential $\delta W(\mathbf{i})$ to the magnetic energy $W(\mathbf{i})$ by Stokes' theorem

$$
\int_{0}^{\mathbf{i}} \delta W(\mathbf{i})=W(\mathbf{i})-W(0)
$$

such that the integral is path independent. By using Eq. (47) and proceeding along the same 1 ines as in Eqs. (39) and (40) we end up with

$$
\begin{aligned}
W(\mathbf{i}) & =\frac{1}{2} \mathbf{i}^{T} \mathbf{L}^{W}(\mathbf{i}) \mathbf{i}+W(0), \\
\mathbf{L}^{W}(\mathbf{i}) & =\int_{0}^{1} 2 \lambda \mathbf{L}(\lambda \mathbf{i}) \mathrm{d} \lambda
\end{aligned}
$$

yet another symmetric inductance matrix which relates the currents to the stored energy. In general $\mathbf{L}(\mathbf{i}) \neq \mathbf{L}^{\psi}(\mathbf{i}) \neq$ $\mathbf{L}^{W}(\mathbf{i})$. This fact is not always adequately emphasised in the literature. Only for linear magnetic systems all three matrices become identical.

\section{Conclusions}

In this paper, some issues arising from the coupling of field and circuit descriptions of electromagnetic devices have been addressed. Not only terminal currents and voltages, but also flux linkages can be unambiguously defined, such that Eq. (28) holds. The determination of the generalised flux linkage follows a simple recipe: 
- Select a pair of terminals which can be connected by a path that lies completely within $\Omega_{\sigma}$.

- Impress a DC current $I_{0}$ and record the resulting normalised DC current density $\underline{\tau}=\underline{J}_{0} / I_{0}$.

- The flux linkage in an arbitrary time-dependent situation is calculated by projecting the vector potential $\underline{A}$ onto $\underline{\tau}$, Eq. (29). In vectorial notation, this becomes

$$
\psi(\boldsymbol{A})=\int_{\Omega_{\sigma}} \boldsymbol{A} \cdot \frac{\boldsymbol{J}_{0}}{I_{0}} \mathrm{~d} \Omega .
$$

To introduce inductances, we have assumed a finite dimensional state space, with the terminal currents as state variables. This can be achieved by admitting filamentary conductors only. In practice, the conductors will have a finite thickness, such that skin and proximity effects occur. These effects have not been included in the definition of the inductances. Such effects would render the inductance matrices dependent not only on the currents, but also on the rate of change of the currents in a complicated manner.

The most basic inductance matrix is the incremental inductance matrix $\mathbf{L}$, whose coefficients are obtained by the rule "derivative of the fluxes w.r.t the currents", Eq. (36). This definition does not rely on the principle of linear superposition. Two other inductance matrices, namely $\mathbf{L}^{\psi}$ and $\mathbf{L}^{W}$, can be constructed by integration, Eqs. (40) and (51), respectively. All inductance matrices are symmetric and, apart from linear magnetic systems, different from each other. The appropriate matrix has to be selected, as the case may be computation of induced voltages, flux linkages or magnetic field energy.

\section{References}

de Rham, G.: Differentiable Manifolds, Springer-Verlag, Berlin, 1984.

Demerdash, N. and Nehl, T.: Electric machinery parameters and torques by current and energy perturbations from field computations - Part I: Theory and formulation, IEEE Transactions on Energy Conversion, 14, 1507-1513, 1999.

Fano, R., Chu, L., and Adler, R.: Electromagnetic Fields, Energy and Forces, John Wiley \& Sons, New York, 1960.

Hantila, F.: Mathematical models of the relation between B and $\mathrm{H}$ for non-linear media, Revue roumaine des sciences techniques Série électrotechnique et énergétique, 19, 429-448, 1974.

Kettunen, L.: Fields and Circuits in Computational Electromagnetism, IEEE Transactions on Magnetics, 37, 3393-3396, 2001.

Munteanu, I. and Ioan, D.: A Survey on Parameter Extraction Techniques for Coupling Electromagnetic Devices to Electric Circuits, in Scientific Computing in Electrical Engineering, edited by U. van Rienen, M. Günther, and D. Hecht, vol. 18 of Lecture Notes in Computational Science and Engineering 337-357, Springer-Verlag, Berlin, 2001.

S. Suuriniemi, J. K. and Kettunen, L.: Detection of State Variables for Coupled Circuit-Field Problems, in Record of the 14th COMPUMAG Conference on the Computation of Electromagnetic Fields, Saratoga Springs, NY, USA, 2003. 